\title{
ADAPTACIÓN Y RENDIMIENTO DE HÍBRIDOS DE ALTA CALIDAD DE PROTEÍNA EN REGIONES TROPICALES Y SUBTROPICALES DE MÉXICO ${ }^{1}$
}

\author{
Ernesto Preciado ${ }^{2}$, Hugo Córdova ${ }^{3}$, Arturo Terrón ${ }^{2}$,Ernesto Cervantes ${ }^{4}$, Esteban Betanzos ${ }^{4}$, Alejandro Ortega ${ }^{4}$, \\ Noel Gómez ${ }^{4}$, Cesar Reyes ${ }^{4}$, Humberto Vallejo ${ }^{4}$, Mauricio Erazo ${ }^{2}$
}

\section{RESUMEN}

\begin{abstract}
Adaptación y rendimiento de híbridos de alta calidad de proteína en regiones tropicales y subtropicales de México. A partir de 1998 se inició un programa nacional de maíz con el fin de desarrollar, identificar y liberar híbridos y variedades de alta calidad de proteína con mayor potencial de rendimiento, adaptados a diversas regiones productoras. En el presente trabajo se predijo el comportamiento de cruzas triples a partir de un análisis conjunto de rendimiento de un dialélico formado con 10 líneas de alta calidad proteínica liberadas por el CIMMYT, que fueron evaluadas en cinco localidades de México durante 1997. Las cruzas triples predichas fueron evaluadas en 11 localidades tropicales y subtropicales de México durante el ciclo primavera-verano 1999. En los resultados obtenidos se observó que algunas cruzas triples predichas produjeron rendimientos similares a las cruzas simples de alta calidad proteínica y a los testigos comerciales normales. Los híbridos que mostraron mayor adaptación a través de localidades involucraron cruzamientos entre líneas tropicales por subtropicales. En regiones tropicales (Iguala, Guerrero) se obtuvieron rendimientos experimentales de hasta de 10 t/ha y en regiones subtropicales (Celaya, Guanajuato) hasta de $16 \mathrm{t} / \mathrm{ha}$. Las cruzas triples predichas constituyen una buena alternativa en países con climas tropicales y subtropicales donde el uso de las cruzas simples, aún no se ha generalizado debido a problemas de producción de semilla, con la ventaja del carácter de alta calidad proteínica para las regiones donde el consumo de maíz es significativo.
\end{abstract}

\begin{abstract}
Adaptation and grain yield of quality protein maize hybrids in Mexican tropical and subtropical regions. A national quality protein maize program was initiated to develop, identify and release high yield potential quality protein maize hybrids and varieties adapted to different regions. Three-way crosses were predicted, based on 10 quality protein maize inbred lines diallel, which were evaluated at five Mexican locations during 1997. The predicted three-way crosses performance were evaluated at 11 tropical and subtropical Mexican locations during 1999. The superior group of predicted three-way crosses showed a good performance in almost all locations. They were competitive in grain yield with quality protein maize single crosses and normal commercial hybrids. The three-way quality protein maize hybrids with the best adaptation across locations were hybrids which involved tropical and subtropical inbred lines. In tropical regions the highest grain yield (10 t/ha) were obtained at Iguala, Guerrero. In subtropical regions the highest grain yield (16 t/ha) were obtained at Celaya, Guanajuato. The predicted three way hybrids are considered a good alternative for countries with tropical and subtropical climate were the use of single crosses is not common yet, because of seed production problems, with the advantage of quality protein maize character for regions where maize consumption is significant.
\end{abstract}

\section{INTRODUCCIÓN}

El maíz de alta calidad de proteína constituye una alternativa para mejorar la alimentación de grandes sectores de población en diversos países latinoamericanos, donde el maíz es una de las más importantes fuentes de carbohidratos y proteínas. En el caso de México el consumo de tortillas de maíz, aporta el 59\% de la ingesta calórica nacional y el 39\% de la ingesta proteínica (Bourges, citado por Turrent, 1994).

\footnotetext{
1 Presentado en la XLVI Reunión Anual del PCCMCA, Puerto Rico, 2000.

2 Programa de Maíz del Campo Experimental Bajío, Apdo. Postal 112, 38000, Celaya, Gto. México. Tel. (52) 461-153-23 ext. 123 // email: preciado@cirpac.inifap.conacyt.mx.

3 Programa Tropical de Maíz del CIMMYT.

4 Programa de Maíz del INIFAP.
} 
Con el descubrimiento del gene opaco 2 del maíz en la década de los sesenta, y con el trabajo visionario del Dr. Vasal y la Dra. Eva Villegas (Lab), de iniciar la selección de genes modificadores en los maíces opacos, se llegó a la obtención de los maíces con alta calidad de proteína (MCP) o QPM (Quality Protein Maize) los cuales mantienen en el endospermo del grano, altos niveles de lisina y triptofano como el maíz opaco, y además pero poseen un endospermo vítreo similar al del maíz normal.

Después de la liberación de una serie de líneas endogámicas con el carácter MCP, descritas por Vasal (1994), el CIMMYT detuvo por un tiempo sus investigaciones en este tipo de maíces. A mediados de los noventa algunas organizaciones no gubernamentales manifestaron su interés por este tipo de materiales por lo que en el CIMMYT, se reinició el trabajo con maíces de MCP, a través de un dialélico entre un grupo de líneas liberadas, buscando la combinación entre líneas de origen tropical con las de origen subtropical. Como resultado se obtuvo una serie de cruzas simples de alto potencial de rendimiento, las cuales de manera competitiva presentaron rendimientos similares o superiores a los híbridos comerciales de endospermo normal de diversas empresas semilleras privadas.

Desafortunadamente el uso de híbridos de maíz bilineales o cruza simple de maíz por productores de diversos países latinoamericanos con climas tropicales y subtropicales es todavía limitado, debido a que en este tipo de híbridos la semilla se produce en una de las dos líneas endogámicas, cuya naturaleza genética se refleja en una menor producción por unidad de superficie. Aunado a lo anterior, existe una capacidad limitada de las pequeñas y medianas empresas semilleras regionales, en manejar grandes superficies de producción. Por estas razones, el costo de producción de semilla de híbridos de cruza simple por kilogramo es mayor, lo cual influye negativamente en los usuarios potenciales de semillas mejoradas, quienes aún no están dispuestos a pagar un sobreprecio por híbridos de dos líneas (Preciado y Terrón, 1997). Otra limitante de la utilización de este tipo de híbridos, está relacionada con la respuesta ambiental de los progenitores, la cual repercute directamente en la producción de los híbridos de cruza simple, que en algunos países como México se realiza en ciclos agrícolas y regiones diferentes al área de adaptación del híbrido.

Con base en la problemática existente en Latinoamérica sobre el uso de híbridos de cruza simple, se planteó como alternativa la formación de cruzas trilineales que satisfagan la demanda de semilla mejorada de maíz en la región, con el valor agregado de mejor calidad poteínica. En este tipo de híbridos se explota la elevada heterosis que aporta el progenitor femenino, la cual es una cruza bilineal, y además la menor interacción con el ambiente, en comparación con las líneas endogámicas utilizadas como progenitores.

En el presente trabajo se predijo el comportamiento de cruzas trilineales de MCP a través del método de Jenkins (1934), con base en la información de un dialélico formado con diez líneas de MCP, con el objetivo de comparar las cruzas trilineales predichas con los rendimientos de campo observados en regiones tropicales y subtropicales de México.

\section{MATERIALES Y MÉTODOS}

Como parte de los trabajos que el CIMMYT realiza en colaboración con el Instituto Nacional de Investigaciones Forestales, Agrícolas y Pecuarias (INIFAP), durante 1997 se condujeron ensayos de rendimiento formados con un dialélico de diez líneas de MCP en cinco localidades de México: Tlaltizapán, Morelos; Celaya, Guanajuato; Pabellón, Aguascalientes; Cd. Obregón, Sonora; y Valle del Fuerte, Sinaloa. En el Cuadro 1, se describen las líneas utilizadas en el dialélico, con el propósito de explotar la heterosis al máximo. Cinco de las líneas son de origen subtropical y cinco de origen tropical.

A partir del análisis conjunto de la información de los ensayos, con las cruzas posibles se procedió a realizar la predicción de cruzas trilineales con base en el método de Jenkins (1934). Para la predicción se utilizaron las medias de rendimiento, obtenidas a través de localidades de las cruzas bilineales del dialélico. Se asumió que las medias a través de las cinco localidades representaron la respuesta genotípica de cada cruza. Las cruzas triples posibles en un dialélico de 10 líneas

Cuadro 1. Genealogía y origen de las líneas de MCP liberadas por el CIMMYT, utilizadas en el dialélico evaluado durante 1997 (Vasal, 1994).

\begin{tabular}{|c|c|c|}
\hline Línea & Genealogía & $\begin{array}{l}\text { Origen del } \\
\text { germoplasma }\end{array}$ \\
\hline CML 173 & $\begin{array}{l}\text { P68c1HC180-1-3-1-1-B-2-B-B } \\
\text { P63-12-2-1/P67-5-1-1)-1-2-B-B }\end{array}$ & Subtropical \\
\hline CML 177 & C32QMH84-2-2-1-1-B-B & Subtropical \\
\hline CML 180 & C32Q/EV8444SRBC4)-\#-B-\#-B-B-21-2-B-B & Subtropical \\
\hline CML 186 & P67c2HC26-1-2-1-B-B & Subtropical \\
\hline CML 140 & P62c3HC87-2-1-\#-\#-1-B-\# & Tropical \\
\hline CML 142 & P62c5HC93-5-6-1-3-B-B-B-7-B-B-\# & Tropical \\
\hline CML 145 & P63c0HC181-3-2-1-4\#-2-B-B-B-B-\#-\# & Tropical \\
\hline CML 146 & АC8563МH35-3-1-B-2-1-B-B-1-B-B-\# & Tropical \\
\hline CML 149 & C24QMH159-2-2-2-B-2-B-B-B-\#-B & Tropical \\
\hline
\end{tabular}


son 360 , es decir $306=1 / 2[n(n-1)(n-2)]=1 / 2[10(9)(8)]$ =360. (Hallauer y Miranda, 1988). La predicción se hizo mediante el programa en Fortran escrito por Crossa (comunicación personal, 1999). De las 360 cruzas trilineales se eligieron las de mayor potencial de rendimiento, para su formación y posterior evaluación.

Las cruzas triples predichas elegidas se formaron durante 1998, y se evaluaron en primavera-verano de 1999 en diversas localidades tropicales y subtropicales del país, en condiciones de riego y temporal (Cuadro 2).

Se considera como un ensayo uniforme al conjunto de experimentos cuyos tratamientos (genotipos) son iguales y son sembrados en diversas localidades.

Los ensayos uniformes establecidos en las regiones tropicales estuvieron integrados por 64 genotipos que pueden ser agrupados de la siguiente forma: a) cruzas trilineales predichas de MCP, b) cruzas simples $\mathrm{y}$ trilineales de MCP superiores en ensayos de evaluación durante 1997 y 1998, y c) testigos comerciales de endospermo normal, provenientes del INIFAP y de empresas semilleras privadas. Los ensayos uniformes establecidos en las regiones subtropicales estuvieron integrados por 49 genotipos agrupados de la siguiente forma: a) cruzas trilineales predichas de MCP, b) cruzas simples y trilineales de MCP superiores en ensayos de evaluación durante 1997 y 1998, y c) testigos comerciales de endospermo normal, provenientes del INIFAP y de empresas semilleras privadas. En ambos tipos de ensayos las cruzas trilineales predichas fueron las mismas. En las localidades tropicales se utilizó un diseño experimental de látice $8 \times 8$ y en las localidades subtropicales fue un látice $7 \times 7$ con dos repeticiones por localidad.

Con la información obtenida se condujo el análisis conjunto de los ensayos tropicales, y subtropicales. A partir de dichos análisis se calcularon los parámetros de estabilidad de las cruzas trilineales predichas, con el objeto de determinar su estabilidad a través de los ambientes tropicales y subtropicales en comparación con los testigos de endospermo normal, utilizando el método de Everhart y Russell (1966).

Los híbridos trilineales mejores de ensayos previos se pusieron a manera de testigos para tener una base de comparación con los híbridos predichos que se están evaluando.

\section{RESULTADOS Y DISCUSIÓN}

En el Cuadro 3 se presentan los rendimientos medios de las cruzas simples que forman el dialélico integrado con 10 líneas de MCP evaluadas durante 1997 en cinco localidades de México. Se puede apreciar que las líneas CML 142, CML 186, CML 176 y CML 149, la primera y la ultima de origen tropical y las otras dos de origen subtropical fueron las que presentaron una media más alta a través de cruzamientos, pudiendo tomar esta media como un valor que representa la aptitud combinatoria general. De hecho, las mejores combinaciones específicas fueron las cruzas de CML 186 x CML 149 y CML 186 x CML 142, con 10,99 y 10,63 t/ha, respectivamente. En ambas cruzas se manifiesta la heterosis entre líneas de origen tropical por subtropical.

A partir de las medias de rendimiento de grano (Cuadro 3) se realizó la predicción de rendimiento de las 360 cruzas triples posibles, de las cuales se eligió el estrato superior en rendimiento representado por 19 cruzas trilineales predichas formadas durante 1998. No hubo un criterio determinado para decir que se evaluarían 19 de las 360 cruzas triples predichas, simplemente del estrato superior de cruzas triples predichas 19 de ellas fueron las que fue posible formar por disponibilidad de semilla, coincidencia en la floración de los progenitores entre otros factores.

En el Cuadro 4 se presenta el rendimiento de grano predicho de las cruzas trilineales de MCP, el rendimiento medio a través de localidades, así como el rendimiento obtenido en cada una de las siete localidades

Cuadro 2. Localidades de evaluación de las cruzas triples predichas y condición de humedad. México. Primavera/verano 1999.

\begin{tabular}{lllc}
\hline $\begin{array}{c}\text { Localidades } \\
\text { Tropicales }\end{array}$ & $\begin{array}{c}\text { Condición de } \\
\text { humedad }\end{array}$ & \multicolumn{1}{c}{$\begin{array}{c}\text { Localidades } \\
\text { Subtropicales }\end{array}$} & $\begin{array}{c}\text { Condición de } \\
\text { humedad }\end{array}$ \\
\hline Iguala, Guerrero & Temporal & Morelia, Michoacán & Riego \\
Ocozocuautla, Chiapas & Temporal & Pabellón, Aguascalientes & Riego \\
Villaflores, Chiapas & Temporal & Celaya, Guanajuato & Riego \\
Sur de Tamaulipas A & Riego & Cortazar, Guanajuato & Riego \\
Sur de Tamaulipas B & Riego & & \\
Río Bravo, Tamaulipas & Riego & & \\
Cd. Obregón, Sonora & Riego & & \\
\hline
\end{tabular}


Cuadro 3. Rendimiento medio en t/ha a través de cinco localidades de las cruzas simples posibles, provenientes de un dialélico entre 10 líneas de MCP liberadas por el CIMMYT (Córdova et al., 1998).

\begin{tabular}{|c|c|c|c|c|c|c|c|c|c|c|}
\hline & CML 173 & CML 176 & CML 177 & CML 180 & CML 186 & CML 140 & CML 142 & CML 145 & CML 146 & CML 149 \\
\hline CML 173 & & 9,04 & 7,19 & 6,89 & 9,07 & 9,10 & 9,99 & 9,04 & 8,86 & 9,79 \\
\hline CML 176 & & & 9,67 & 9,12 & 10,11 & 9,17 & 9,62 & 8,51 & 8,04 & 8,77 \\
\hline CML 177 & & & & 6,91 & 8,92 & 7,81 & 8,99 & 7,42 & 8,77 & 8,93 \\
\hline CML 180 & & & & & 8,31 & 7,17 & 9,22 & 7,09 & 9,56 & 8,66 \\
\hline CML 186 & & & & & & 8,07 & 10,63 & 8,61 & 9,32 & 10,99 \\
\hline CML 140 & & & & & & & 8,63 & 8,40 & 8,72 & 8,42 \\
\hline CML 142 & & & & & & & & 9,69 & 9,59 & 9,04 \\
\hline CML 145 & & & & & & & & & 7,43 & 7,44 \\
\hline CML 146 & & & & & & & & & & 8,34 \\
\hline CML 149 & & & & & & & & & & \\
\hline Media & 8,77 & 9,12 & 8,29 & 8,10 & 9,34 & 8,38 & 9,48 & 8,18 & 8,73 & 8,93 \\
\hline
\end{tabular}

tropicales de evaluación durante el ciclo agrícola de primavera-verano 1999. Se puede observar que el rendimiento predicho de las cruzas trilineales de MCP ordenado de mayor a menor presenta una variación que va de 10,7 a $9,7 \mathrm{t} / \mathrm{ha}$, los cuales se encuentran dentro del mismo intervalo de rendimiento de las cruzas simples superiores del Cuadro 3. Debido a que son rendimientos medios obtenidos en 1997 en cinco localidades, no se tiene un punto de comparación directo con los rendimientos a través de las localidades tropicales mostrados en el Cuadro 4. Sin embargo, es posible ilustrar el comportamiento de las cruzas trilineales predichas en comparación con cada una de las localidades tropicales.
Las cruzas triples predichas que mostraron rendimiento medio mayor a través de las localidades tropicales fueron (CML 186 x CML 142) x CML 176, (CML 142 x CML 146) x CML 186 y (CML 176 x CML 186) x CML 142, con 6,85; 6,82 y 6,78 t/ha, respectivamente. Como se puede observar en estas cruzas intervienen las líneas de mejor aptitud combinatoria que fueron presentadas en el Cuadro 3.

En general se puede observar que un buen número de cruzas trilineales predichas presentó rendimientos superiores a la media de cada experimento, y en algunos casos existen híbridos predichos iguales o superio-

Cuadro 4. Medias de rendimiento ( $\mathrm{t} / \mathrm{ha}$ ) de las cruzas triples de MCP predichas por localidad y a través de localidades tropicales de México. Primavera/verano, 1999.

\begin{tabular}{|c|c|c|c|c|c|c|c|c|c|}
\hline \multirow[t]{2}{*}{ Híbrido } & \multicolumn{9}{|c|}{ Rendimiento (t/ha) } \\
\hline & Predicho & $\begin{array}{c}\text { Media } \\
\text { Tropical }\end{array}$ & $\begin{array}{l}\text { Villa } \\
\text { Flores }\end{array}$ & Ocoz. & $\begin{array}{c}\text { Sur } \\
\text { Tam A }\end{array}$ & $\begin{array}{c}\text { Rio } \\
\text { Bravo }\end{array}$ & Iguala & $\begin{array}{c}\text { Sur } \\
\text { Tam B }\end{array}$ & Obregón \\
\hline (CML 142 X CML 149) X CML 186 & 10,7 & 6,30 & 4,13 & 7,27 & 4,83 & 4,65 & 7,54 & 4,78 & 10,89 \\
\hline (CML 176 X CML 149) X CML 186 & 10,5 & 6,10 & 3,42 & 7,12 & 3,93 & 3,72 & 9,52 & 4,43 & 10,56 \\
\hline (CML 176 X CML 142) X CML 186 & 10,4 & 6,25 & 2,76 & 6,95 & 5,13 & 4,80 & 8,08 & 5,44 & 10,56 \\
\hline (CML 173 X CML 186) X CML 142 & 10,3 & 6,39 & 3,86 & 7,65 & 5,68 & 4,88 & 9,08 & 4,36 & 9,20 \\
\hline (CML 186 X CML 145) X CML 142 & 10,2 & 6,19 & 3,84 & 8,24 & 5,05 & 4,13 & 9,10 & 3,98 & 9,01 \\
\hline (CML 176 X CML 186) X CML 142 & 10,1 & 6,78 & 3,14 & 7,80 & 6,85 & 4,40 & 8,70 & 5,48 & 11,09 \\
\hline (CML 142 X CML 146) X CML 186 & 10,0 & 6,82 & 4,04 & 7,82 & 5,43 & 4,74 & 9,92 & 4,85 & 10,94 \\
\hline (CML 173 X CML 142) X CML 186 & 9,9 & 6,73 & 4,64 & 7,67 & 5,53 & 4,52 & 9,42 & 4,80 & 10,55 \\
\hline (CML 177 X CML 186) X CML 149 & 9,9 & 5,14 & 3,94 & 6,60 & 3,20 & 2,87 & 8,33 & 3,00 & 8,02 \\
\hline (CML 177 X CML 149) X CML 186 & 9,9 & 5,44 & 2,74 & 6,17 & 3,25 & 4,06 & 7,92 & 3,20 & 10,75 \\
\hline (CML 180 X CML 186) X CML 142 & 9,9 & 6,31 & 4,46 & 7,18 & 5,60 & 4,37 & 8,13 & 4,90 & 9,57 \\
\hline (CML 186 X CML 142) X CML 176 & 9,9 & 6,85 & 3,37 & 7,30 & 6,35 & 4,75 & 9,53 & 5,89 & 10,73 \\
\hline (CML 186 X CML 142) X CML 149 & 9,9 & 6,24 & 4,53 & 7,95 & 5,20 & 4,00 & 7,88 & 4,20 & 9,90 \\
\hline (CML 142 X CML 149) X CML 173 & 9,9 & 5,45 & 3,28 & 6,33 & 4,42 & 4,21 & 7,93 & 4,48 & 7,51 \\
\hline (CML 173 X CML 145) X CML 142 & 9,8 & 5,28 & 3,88 & 6,58 & 4,70 & 3,54 & 7,98 & 2,70 & 7,55 \\
\hline (CML 176 X CML 186) X CML 149 & 9,8 & 6,30 & 4,97 & 7,61 & 4,10 & 3,38 & 9,04 & 3,83 & 11,15 \\
\hline (CML 177 X CML 186) X CML 142 & 9,8 & 6,31 & 4,47 & 5,65 & 5,65 & 3,87 & 9,86 & 3,59 & 11,08 \\
\hline (CML 180 X CML 186) X CML 149 & 9,8 & 6,01 & 4,25 & 7,09 & 3,13 & 3,48 & 9,62 & 5,14 & 9,35 \\
\hline (CML 145 X CML 176) X CML 142 & 9,7 & 6,26 & 3,41 & 6,54 & 5,65 & 3,89 & 9,03 & 3,94 & 11,38 \\
\hline H $365 \mathrm{C}$ & & 6,75 & 3,98 & 7,57 & 5,73 & 4,73 & 9,27 & 4,60 & 11,41 \\
\hline H $553 \mathrm{C}$ & & 7,28 & 4,01 & 8,63 & 7,23 & 3,80 & 8,85 & 5,23 & 13,15 \\
\hline Medias & & 5,99 & 3,52 & 7,34 & 4,99 & 3,60 & 8,64 & 3,80 & 10,06 \\
\hline LSD 0,05 & & 0,22 & 0,45 & 0,63 & 0,52 & 0,28 & 0,77 & 0,51 & 0,61 \\
\hline C.V.(\%) & & 13,73 & 18,75 & 12,77 & 15,14 & 10,28 & 11,54 & 19,43 & 8,80 \\
\hline
\end{tabular}


res estadísticamente a los testigos $\mathrm{H} 365 \mathrm{C}$ y $\mathrm{H} 553 \mathrm{C}$, que son cruzas trilineales de MCP superiores a través de años en varias localidades de México. Sin embargo, es importante reconocer el excelente comportamiento del H553C a través de las localidades tropicales.

Por otro lado, a través de las medias por experimento, se puede apreciar que las localidades tropicales donde los materiales expresaron un mayor potencial de rendimiento, fueron Obregón, Iguala y Ocozocuautla.

En el Cuadro 5 se presenta el rendimiento de grano predicho de las cruzas trilineales de MCP, el rendimiento medio a través de localidades, así como el rendimiento obtenido en cada una de las cuatro localidades subtropicales durante el ciclo agrícola primavera-verano 1999. La información del rendimiento predicho de las cruzas trilineales de MCP ilustra el comportamiento de las cruzas trilineales predichas en comparación con cada una de las localidades subtropicales. En este caso, los rendimientos predichos son más comparables, que en el caso de las localidades tropicales cuyo potencial de rendimiento es menor.

Las cruzas trilineales predichas que mostraron rendimiento medio mayor a través de las localidades subtropicales fueron (CML 176 x CML 186) x CML 142,
(CML 186 x CML 145) x CML 142, y (CML 142 x CML 146) x CML 186, con $11,65,11,60$ y 11,10t/ha, respectivamente. Es importante resaltar que tanto la primera como la tercer cruza, pero en posición diferente, fueron también las superiores a través de las localidades tropicales. Con estos datos se resalta la importancia de las combinaciones heteróticas de material tropical $\mathrm{x}$ subtropical sobre todo para ambientes de subtrópico.

En el caso de las regiones trilineales subtropicales se observó un mayor número de cruzas predichas que presentaron rendimientos superiores a la media de cada experimento por localidad, y también es notorio un mayor número de casos donde los híbridos predichos igualan o superan estadísticamente a los testigos H365C y $\mathrm{H} 553 \mathrm{C}$, que son cruzas trilineales de MCP superiores a través de años en varias localidades de México. En este caso el híbrido H365C fue el testigo con mejor comportamiento a través de las localidades subtropicales.

Al comparar las medias por experimento, se puede apreciar que Celaya, Guanajuato fue la localidad subtropical donde los materiales expresaron un mayor potencial de rendimiento.

Recapitulando sobre la información presentada en los Cuadros 4 y 5 , respecto a los rendimientos de las

Cuadro 5. Medias de rendimiento ( $\mathrm{t} / \mathrm{ha}$ ) de las cruzas trilineales de MCP predichas por localidad y a través de localidades subtropicales de México. Primavera-verano 1999

\begin{tabular}{|c|c|c|c|c|c|c|}
\hline \multirow[t]{2}{*}{ Hibrido } & \multicolumn{6}{|c|}{ Rendimiento (t/ha) } \\
\hline & Predicho & $\begin{array}{c}\text { Media } \\
\text { Subtropical }\end{array}$ & Morelia & Pabellón. & Celaya & Cortazar \\
\hline $\begin{array}{l}\text { (CML } 142 \text { X CML 149) X CML } 186 \\
\text { (CML } 176 \text { X CML 149) X CML } 186\end{array}$ & $\begin{array}{l}10,7 \\
10,5\end{array}$ & $\begin{array}{r}9,97 \\
10,97\end{array}$ & $\begin{array}{l}8,05 \\
9,22\end{array}$ & $\begin{array}{r}8,93 \\
10,79\end{array}$ & $\begin{array}{l}13,06 \\
14,71\end{array}$ & $\begin{array}{l}9,83 \\
9,16\end{array}$ \\
\hline (CML 176 X CML 142) X CML 186 & 10,4 & 11,07 & 7,15 & 11,35 & 14,98 & 10,79 \\
\hline (CML 173 X CML 186) X CML 142 & 10,3 & 10,06 & 7,53 & 9,91 & 13,63 & 9,18 \\
\hline (CML 186 X CML 145) X CML 142 & 10,2 & 11,60 & 11,96 & 10,29 & 14,94 & 9,22 \\
\hline (CML 176 X CML 186) X CML 142 & 10,1 & 11,65 & 10,38 & 10,42 & 16,03 & 9,78 \\
\hline (CML 142 X CML 146) X CML 186 & 10,0 & 11,10 & 10,26 & 10,72 & 14,70 & 8,72 \\
\hline (CML 173 X CML 142) X CML 186 & 9,9 & 10,82 & 11,50 & 9,81 & 12,40 & 9,59 \\
\hline (CML 177 X CML 186) X CML 149 & 9,9 & 9,30 & 9,04 & 9,01 & 13,40 & 5,74 \\
\hline (CML 177 X CML 149) X CML 186 & 9,9 & 10,87 & 9,81 & 10,96 & 13,96 & 8,74 \\
\hline (CML 180 X CML 186) X CML 142 & 9,9 & 10,97 & 9,91 & 9,11 & 13,93 & 10,91 \\
\hline (CML 186 X CML 142) X CML 176 & 9,9 & 10,32 & 7,38 & 9,76 & 13,83 & 10,31 \\
\hline (CML 186 X CML 142) X CML 149 & 9,9 & 9,83 & 8,03 & 8,96 & 14,27 & 8,04 \\
\hline (CML 142 X CML 149) X CML 173 & 9,9 & 10,36 & 6,99 & 10,85 & 14,65 & 8,95 \\
\hline (CML 173 X CML 145) X CML 142 & 9,8 & 10,77 & 12,03 & 8,93 & 13,46 & 8,63 \\
\hline (CML 176 X CML 186) X CML 149 & 9,8 & 10,46 & 7,70 & 9,60 & 13,80 & 10,75 \\
\hline (CML 177 X CML 186) X CML 142 & 9,8 & 10,02 & 8,15 & 9,59 & 13,98 & 8,36 \\
\hline (CML 180 X CML 186) X CML 149 & 9,8 & 10,21 & 10,75 & 9,60 & 13,07 & 7,40 \\
\hline (CML 145 X CML 176) X CML 142 & 9,7 & 10,63 & 7,51 & 11,54 & 14,89 & 8,57 \\
\hline $\mathrm{H} 365 \mathrm{C}$ & & 10,80 & 9,88 & 10,38 & 14,24 & 8,70 \\
\hline $\mathrm{H} 553 \mathrm{C}$ & & 9,66 & 8,43 & 8,30 & 13,00 & 8,93 \\
\hline Medias & & 10,21 & $\mathbf{9 , 1 0}$ & $\mathbf{9 , 4 3}$ & 13,30 & $\mathbf{9 , 0 3}$ \\
\hline LSD 0,05 & & $\mathbf{0 , 4 8}$ & $\mathbf{1 , 0 3}$ & $\mathbf{0 , 5 9}$ & $\mathbf{0 , 9 0}$ & 0,91 \\
\hline C.V. $(\%)$ & & 13,36 & 15,12 & 8,45 & 8,50 & 12,48 \\
\hline
\end{tabular}


cruzas trilineales de MCP predichas con base en los datos de 1997, en comparación con el comportamiento observado en cada una de las localidades tropicales y subtropicales, es importante recalcar, que la predicción del rendimiento no necesariamente obedece a la adaptación de las mismas a través de localidades. Por lo tanto para estimar esta adaptación se precedió a realizar parámetros de estabilidad por el método de Eberhart y Russell (1966).

En la Figura 1 se presentan las líneas de regresión a través de los índices ambientales de algunas cruzas trilineales predichas de MCP en comparación con los testigos H365C y H553C, a través de las siete localidades tropicales de México, durante 1999. En general los híbridos predichos de cruza triple que se presentan en esta gráfica, fueron algunos de los que presentaron los mejores valores de adaptación a través de localidades, solamente llama la atención la cruza (CML 180 x CML 186) x CML 142 que presenta una interacción donde su comportamiento es mejor en ambientes malos ambientes que en buenos. También se puede observar que el testigo $\mathrm{H} 553 \mathrm{C}$ en los mejores ambientes de las localidades tropicales, expresó su mayor potencial de rendimiento y en general presenta una buena adaptación a través de las localidades tropicales.

En la Figura 2 se presentan las líneas de regresión a través de los índices ambientales de algunas cruzas trilineales predichas de $\mathrm{MCP}$ en comparación con los testigos H365C y H553C, a través de las cuatro localidades de evaluación subtropicales de México durante 1999. El genotipo que a través de ambientes presenta

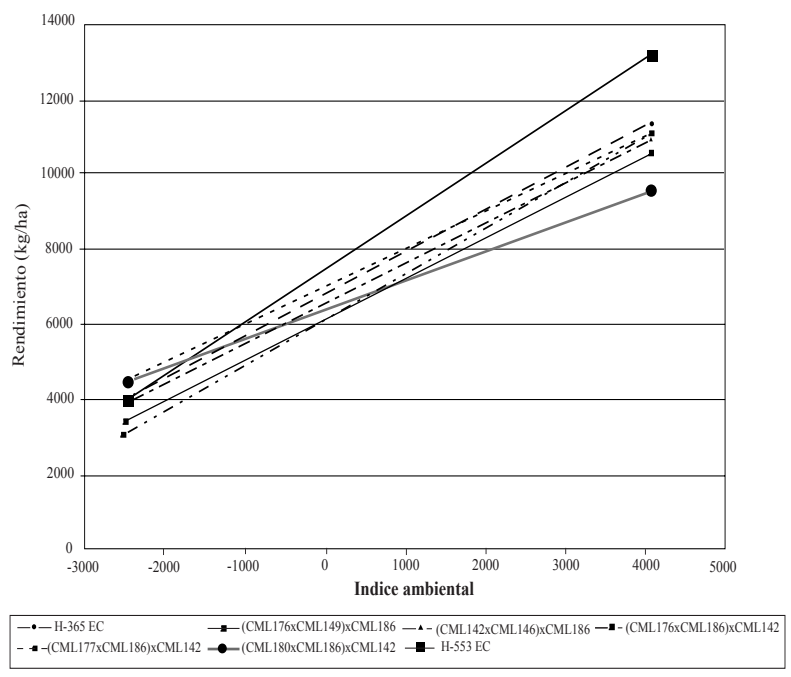

Figura 1. Comportamiento a través de los índices ambientales de algunas cruzas triples predichas de QPM en comparación con los testigos H365C y H553C, a través de siete localidades tropicales de México. Primavera/verano 1999.

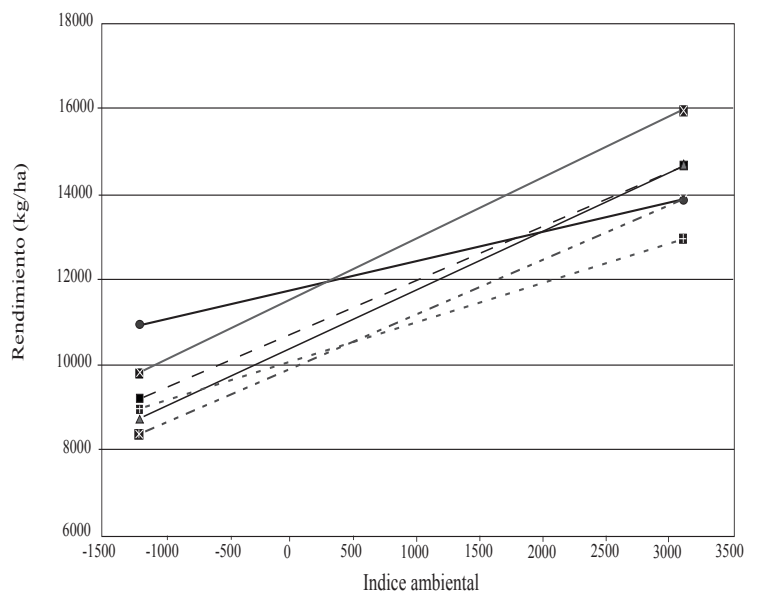

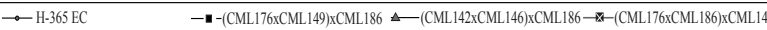
$-\mathbb{W}-($ CML177xCML186)xCML142 - - (CML180xCML186)xCML142 - - H-553 EC

Figura 2. Comportamiento a través de los índices ambientales de algunas cruzas triples predichas de QPM en comparación con los testigos H365C y H553C, a través de cuatro localidades subtropicales de México. Primavera/verano 1999.

una muy buena estabilidad y mayor rendimiento a través de las localidades subtropicales es el híbrido predicho (CML 176 x CML 186) x CML 142, y fue uno de los mejores híbridos tanto en localidades subtropicales como tropicales. En general el resto de los híbridos predichos de cruza triple que se presentan en esta gráfica, fueron algunos de los que presentaron los mejores valores de adaptación a través de localidades. Llama la atención la cruza (CML 180 x CML 186) x CML 142 cuyo comportamiento fue mejor en ambientes malos que en buenos. El comportamiento del testigo H553C fue inferior al de los materiales incluidos en dicha figura, en los mejores ambientes de las localidades subtropicales.

A manera de conclusión se puede comentar que las cruzas trilineales predichas constituyen una buena alternativa en países con climas tropicales y subtropicales, donde el uso de las cruzas simples aún no se ha generalizado debido a problemas de producción de semilla, con la ventaja del carácter MCP para las regiones donde el consumo de maíz es significativo.

\section{LITERATURA CITADA}

CÓRDOVA, H.S.; CASTELLANOS, S.; LÓPEZ, R. 1998. Annual research report 1997. Subtropical maize Subprogram. CIMMYT. $460 \mathrm{p}$.

EBERHART, S.A.; RUSSELL, W.A. 1966. Stability parameters in comparing varieties. Crop Sci. 6: 36-40 
HALLAUER, A.R.; MIRANDA, FO., B.J. 1988. Quantitative genetics in maize breeding. USA, Iowa State University Press. 468 p.

JENKINS, M.T. 1934. Methods of estimating the performance of double crosses in corn. J. Am. Soc. Agron. 26: 199-204.

PRECIADO, E.; TERRÓN, A. 1997. Uso potencial de cruzas simples emparentadas de ciclo vegetativo intermedio, para regiones subtropicales de México. Agronomía Mesoamericana $8(2)$ : $72-77$.
TURRENT, A. 1994. Plan de investigación del sistema maíz-tortilla en la Región Centro. Secretaria de Agricultura y Recursos Hidráulicos, Instituto Nacional de Investigaciones Forestales y Agropecuarias. Chapingo, Méx. 55 p.

VASAL, S. 1994. High quality protein corn. In Hallauer, A. ed. High quality protein corn. Florida, USA, CRC Inc. p. 79-122. 